\title{
IMPLEMENTACIÓN DEL MODELO TRANSIT-ORIENTED DEVELOPMENT (T.O.D.) EN LA CIUDAD DE ALICANTE
}

\author{
Héctor Fernández Herráiz \\ Ingeniero de Caminos, Canales y Puertos, Universidad de Alicante, España \\ Juan Carlos Sánchez Galiano \\ Profesor Asociado, Departamento de Edificación y Urbanismo, Universidad de Alicante, \\ España \\ Patricia Fernández Aracil \\ Investigadora predoctoral, Universidad de Alicante, España \\ Armando Ortuño Padilla \\ Profesor Contratado Doctor, Departamento de Edificación y Urbanismo, Universidad de \\ Alicante, España
}

\section{RESUMEN}

Con el objetivo de combatir los efectos negativos causados por los desarrollos urbanos dispersos o en baja densidad, así como los derivados de la excesiva utilización del vehículo privado para los desplazamientos diarios en la ciudad de Alicante, en el presente artículo se describe un ejemplo de aplicación práctica de implantación del modelo Transit-Oriented Development (TOD) desde una perspectiva integral. Para ello, se considera la fusión de diversos aspectos metodológicos, que resultan complementarios durante la elaboración del estudio y se exponen brevemente en las siguientes líneas.

En primer lugar, mediante el análisis de resultados del estudio de movilidad, efectuado previamente, a propósito de este estudio, es posible averiguar el área de influencia de las paradas de la línea 2 del TRAM (sistema de tranvitrén existente en Alicante) comprendidas en el municipio de Alicante, ámbito al cual se ciñe este proceso de implementación de TOD. En segundo lugar, la recopilación de diferentes variables de bases de datos específicas permite plasmar matemática y gráficamente el modelo Node-Place, mediante el cual es posible caracterizar el entorno de las citadas paradas de TRAM, así como clasificarlas atendiendo al cálculo de dos tipos de indicadores: de nodo, relativos a accesibilidad y a los medios de transporte presentes en el área de influencia, y de lugar, relativos a la diversidad e intensidad de los usos del suelo cercanos a cada estación.

Por último, se presentan las propuestas de actuación que servirían para materializar el modelo TOD en la ciudad en una estación en concreto: aquella que demuestra tener más potencialidad para ello tras analizar el modelo Node-Place. Las medidas de desarrollo urbanístico que promueve el modelo TOD se focalizan en: potenciar el uso del transporte público, densidades de viviendas medias y altas, mezcla de usos urbanos y calles paseables. 


\section{INTRODUCCIÓN}

El desarrollo orientado al transporte público o Transit-Oriented Development (TOD, en adelante) consiste en fomentar un desarrollo urbano, comprendido en un radio de entre 400 y 800 metros de una parada de transporte público, caracterizado por: zonas de viviendas de densidad media-alta, variedad de tipologías habitacionales, mezcla de usos del suelo, accesibilidad mejorada para peatones y ciclistas, menores exigencias de aparcamiento que desarrollos convencionales, transporte público eficiente o viviendas asequibles, entre otras. Este modelo urbanístico surge, en gran parte, como contrapartida a los problemas originados por el proceso conocido como urban sprawl, que consiste en un patrón de desarrollo urbano en baja densidad y disperso en el territorio.

En Curtis et al. (2009), Gibson y Abbott (2002), Knowles (2012) y Ortuño (2013), pueden leerse algunos casos de estudio, tales como: Curitiba (Brasil), Tokyo (Japón), Stedenbaan (Países Bajos), Copenhague (Dinamarca) o EE. UU. En este último país, el TOD es especialmente relevante, por lo que se creó el Center for Transit-Oriented Development, una organización dedicada a proporcionar mejores prácticas de investigación y herramientas para apoyar desarrollos comerciales en comunidades transitables cercanas al transporte público (Center for Transit-Oriented Development, 2009).

En el presente estudio, se esbozan aquellas propuestas de actuación acordes con el TOD en una parada de TRAM de la ciudad de Alicante, considerando aquella que demuestra tener mayor potencialidad, entre todas las paradas analizadas, sirviéndose de un modelo matemático denominado Node-Place. A tal efecto, se precisa, en primer lugar, efectuar estudio de movilidad, que posibilite establecer el área de influencia de las paradas de la Línea 2 del TRAM en el término municipal de Alicante.

\section{2. ÁREA DE ESTUDIO}

El ámbito geográfico es la ciudad de Alicante, analizando específicamente al área de influencia de las once paradas de la Línea 2 del TRAM (L2, en adelante) incluidas en el término municipal de Alicante: desde Luceros hasta Ciudad Jardín, ambas inclusive. La Línea 2 (Fig. 1), en funcionamiento desde el año 2013, discurre entre el centro urbano de Alicante y la zona sur del casco urbano de Sant Vicent del Raspeig. Forma parte de una red tranviaria compuesta por otras líneas urbanas-interurbanas que conectan el centro de Alicante con su entorno metropolitano, así como con diversos municipios de la costa situados al norte de Alicante, entre ellos, Benidorm, Altea o Denia.

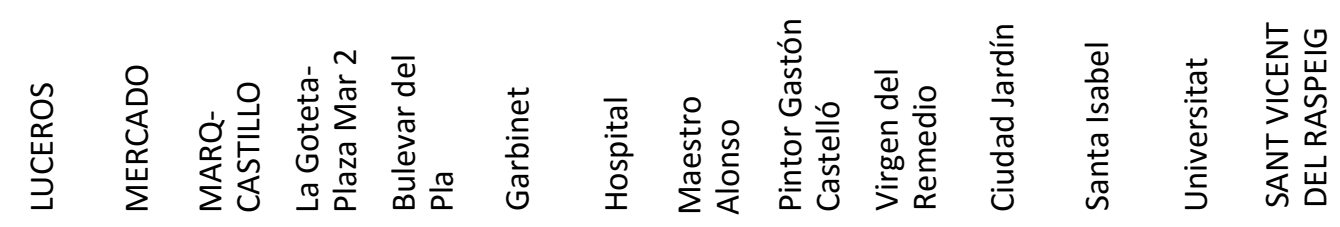




\section{Fig. 1 - Diagrama de la Línea 2}

\section{ESTUDIO DE MOVILIDAD}

En el entorno de cada parada de TRAM —en un radio no superior a $1.000 \mathrm{~m}$, considerando trabajos precedentes (City and County of Denver, 2006) - se efectuaron encuestas de movilidad a individuos de edad igual o superior a 16 años, con el objetivo de obtener el radio de influencia real de las mismas, mediante muestreo aleatorio simple. El tamaño muestral mínimo representativo se corresponde con un total de 330 encuestados. El modelo de formulario para la encuesta se fundamentó en Ibeas et al. (2007), con algunos matices propios de la contextualización para el caso de estudio, incluyendo además cuestiones relativas a la vivienda, la composición del hogar, la actividad económica y la movilidad.

Atendiendo a los resultados obtenidos a partir de las encuestas (Fig. 2), relativos al número de usuarios de TRAM con respecto al total de residentes en el área de influencia de la estación y considerando un nivel de confianza del $95 \%$, el radio de influencia en el presente estudio se corresponde con $500 \mathrm{~m}$.

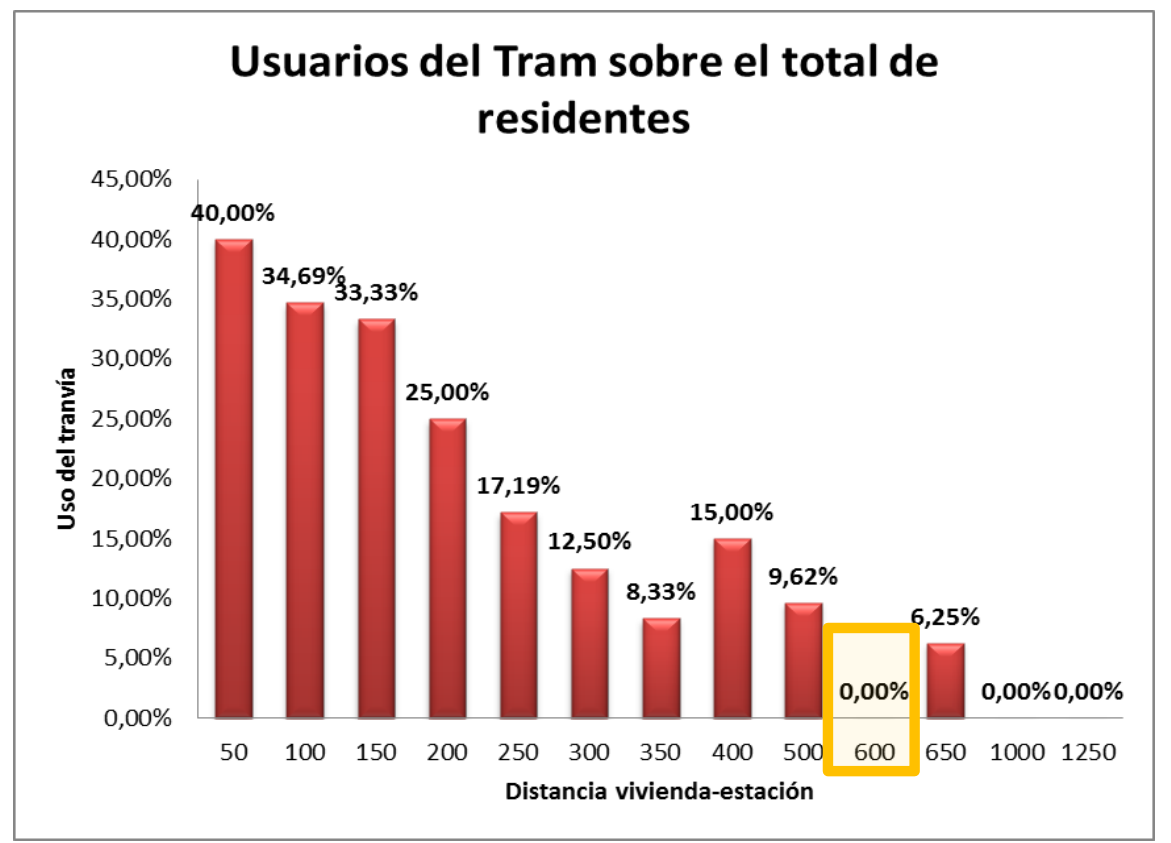

Fig. 2 - Utilización del tranvía en función de la distancia entre la vivienda y la parada

\section{MODELO NODE-PLACE}

El modelo Node-Place permite plasmar matemáticamente las realidades que engloba una estación, pues es: un espacio en la ciudad, un sistema funcional y un foco económico y social. En consonancia con Bertolini (1999), se cuantifican dos aspectos sintéticos de una estación:

- Función de nodo: se corresponde a la accesibilidad del nodo y con el potencial que presenta para la interacción humana física, es decir, cuanta más gente pueda llegar, más interacción será posible. En el presente modelo se dio más importancia a la movilidad urbana y metropolitana frente a la interurbana. 
- Función de lugar: se corresponde con la intensidad y diversidad de las actividades que hay en el lugar y al grado de realización del potencial para la interacción humana, es decir, cuantas más actividades, más interacción estará teniendo lugar.

El estado de la función de nodo y de lugar se obtiene mediante la recopilación y el tratamiento de una serie de variables (indicadores) que sirven para cuantificar el desarrollo de cada función: nodo y lugar. Los resultados obtenidos se transforman en los índices NodePlace, mediante la combinación de dichas variables estableciendo una misma ponderación para cada una. El resultado obtenido se materializa en una nube de puntos sobre una gráfica como la de la Fig. 3.

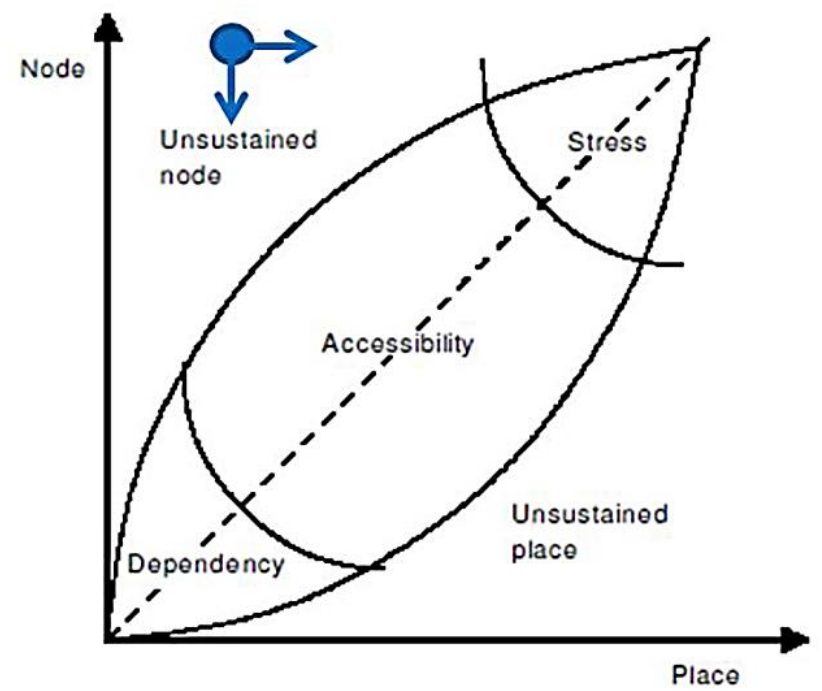

Fig. 3 - Modelo Node-Place. Fuente: Bertolini (1999)

En función de los resultados obtenidos, cada parada de TRAM podría ubicarse en alguna de las cinco situaciones ideales distintas, delimitadas por las áreas en las cuales se divide la gráfica: dependencia, estrés, accesibilidad, nodo insostenible y lugar insostenible. La selección de las variables incluidas en el modelo se ha fundamentado en Bertolini (1999) y Reusser et al. (2008), sin perder de vista la contextualización a este caso de estudio en concreto, con paradas de tranvía en medio urbano, así como la disponibilidad de datos. Por lo tanto, los indicadores seleccionados finalmente se exponen en las Fig. 4 y 5.
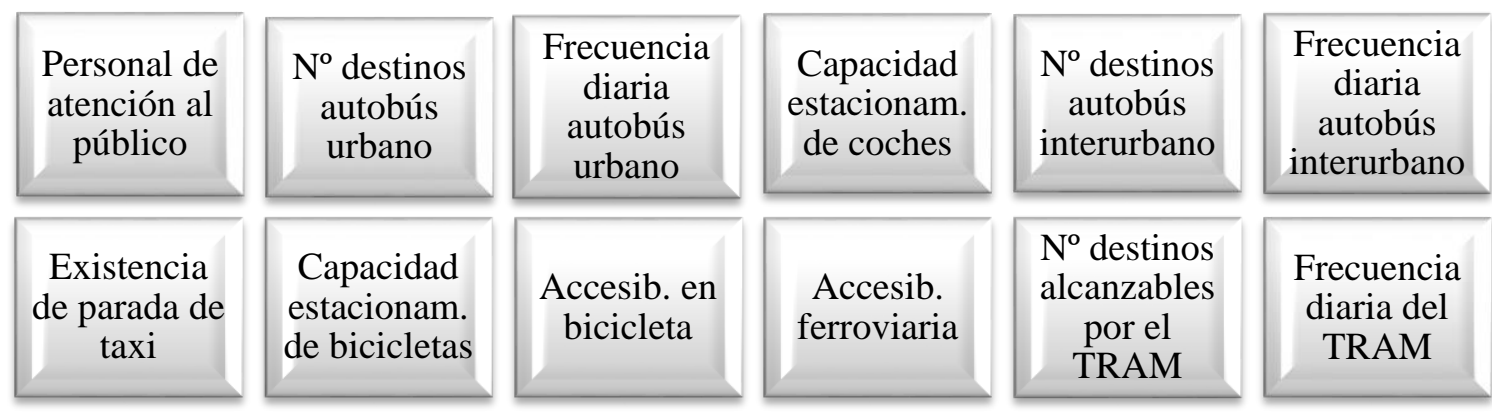

Fig. 4 - Indicadores de nodo 

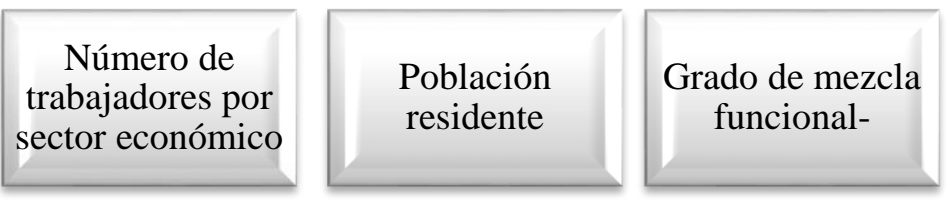

\section{Fig. 5 - Indicadores de lugar}

\section{RESULTADOS Y DISCUSIÓN}

Una vez obtenidos los valores de cada uno de los indicadores y realizado el tratamiento de los datos pertinente, los índices nodo y lugar definitivos (reescalados entre 0 y 1 ) constituyen el modelo Node-Place (Fig. 6). De la gráfica se deduce la situación en la cual se encuentra cada una de las paradas, lo que determina además sus fortalezas y debilidades (Tabla 1).

\begin{tabular}{|l|l|}
\hline Situación ideal & Estación \\
\hline Accesibilidad & Ciudad Jardín, Hospital, Marq \\
\hline Dependencia & Virgen del Remedio, La Goteta \\
\hline Estrés & Marq, Mercado, Luceros \\
\hline Nodo insostenible & Gastón Castelló, Virgen del Remedio, Maestro Alonso \\
\hline Lugar insostenible & Garbinet, Bulevar del Plà, Luceros \\
\hline
\end{tabular}

Tabla 1 - Situación Node-Place de cada parada de TRAM

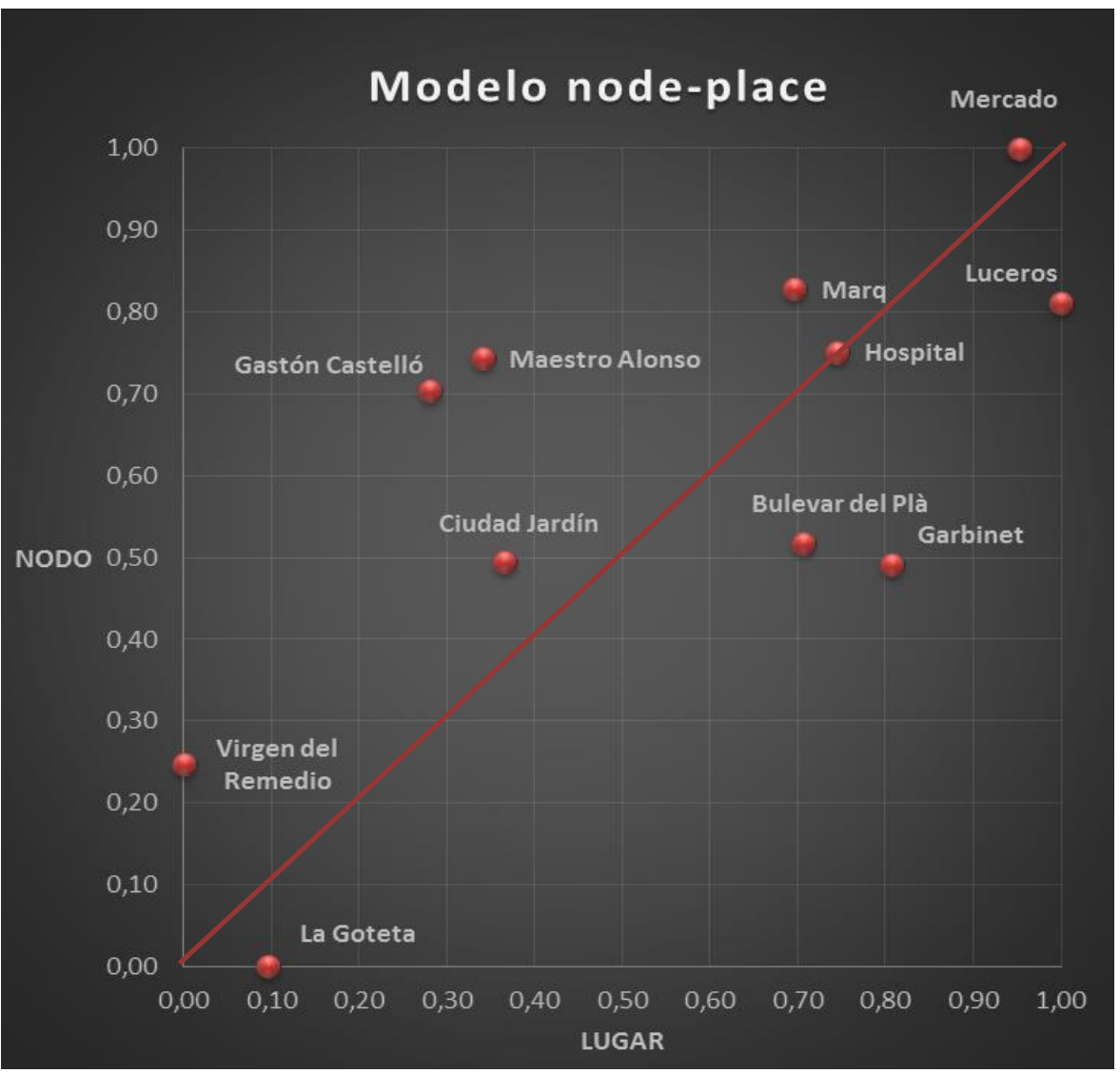

Fig. 6 - Representación del modelo Node-Place para la L2 del TRAM en Alicante 
Considerando que las propuestas de actuación deben focalizarse en la función lugar — puesto que, debido a condicionantes técnicos relativos a la capacidad tranviaria de la infraestructura, en la actualidad no es posible plantear cambios sobre la función nodo-, las estaciones con mayor potencialidad en la función lugar, resultan: Virgen del Remedio, Gastón Castelló y Maestro Alonso. Estas tres áreas, además, están caracterizadas por un bajo nivel de renta, presencia de grupos en riesgo de exclusión social y reducido nivel educativo respecto al resto del municipio, así como bajas tasas de actividad económica en comparación con el nivel medio de la ciudad (Ayuntamiento de Alicante, 2007). Estas características hacen idónea la implementación de un modelo TOD que pueda servir como motor de cambio del área en cuestión.

Otro de los requisitos para llevar a cabo una implementación del modelo TOD satisfactoria es que existan suficientes parcelas no urbanizadas, próximas entre sí, preferiblemente; dicho condicionante es utilizado con éxito en EE. UU., denominado concentración parcelaria (Reconnecting America, 2009). Puesto que Gastón Castelló no cumple con este requisito, queda descartada. En el caso de Virgen del Remedio, se advierte en su tejido urbano una concentración de parcelas desocupadas al norte del área de influencia, así como una densificación de viviendas al sur, por lo que serían precisas propuestas urbanísticas de mayor calado si el objetivo es resolver los problemas intrínsecos de su estructura urbana. Por todo lo anteriormente expuesto, la parada seleccionada para la aplicación del modelo TOD es Maestro Alonso.

\section{CONCLUSIÓN E IMPLEMENTACIÓN DEL MODELO TOD}

Con el objetivo de extraer las propuestas de actuación acordes con el entorno urbano seleccionado, primero es preciso examinar los usos del suelo actuales, así como la dotación de equipamientos y servicios existentes, barriendo un radio de $500 \mathrm{~m}$ en torno a la parada. Las fuentes empleadas con este objetivo son: el portal web de consulta de la encuesta de infraestructura y equipamientos locales (2015), a través de la Diputación de Alicante, y la operadora de transporte público de la ciudad de Alicante, Vectalia Subus (2015). Las deficiencias urbanas de cada zona se determinaron en sintonía con los indicadores de sostenibilidad del Ministerio de Agricultura, Alimentación y Medio Ambiente (2010), mediante los cuales se estableció el umbral mínimo de equipamientos para cumplir con un grado de cobertura suficiente.

Seguidamente se procedió a catalogar aquellas zonas desocupadas o sin uso actual, determinando su ubicación, dimensiones y características, sobre las cuales se propone un nuevo desarrollo urbanístico, fusionando las deficiencias detectadas con los principios del TOD. Maestro Alonso, específicamente, se caracteriza por una escasa fuerza del mercado laboral, débil mezcla de usos y riesgo de sobredensificación. Este último parámetro, correspondiente a 88 viviendas por hectárea, determina que el área quede clasificada como de alta densidad según la nueva Ley de Ordenación del Territorio, Urbanismo y Paisaje de la Comunitat Valenciana, razón por la cual no es recomendable edificar más viviendas. 
Por lo tanto, seguidamente se resumen las propuestas de actuación urbanística que responden a las carencias detectadas, así como al cumplimiento de los principios del TOD:

- Mercado municipal. Debido a la ausencia de mercados que den cobertura a esta zona de la ciudad, y a la demanda vecinal preexistente y para contribuir a la creación de empleo y la mezcla de usos.

- Complejo recreativo formado por complejo lúdico-recreativo y zona de restaurantes.

- Colegio de primaria y educación infantil público. Por la insuficiente dotación existente en la zona suroeste del área de influencia y así lograr una mayor mezcla de usos.

- Instalaciones deportivas públicas. En esta zona del área hay peor acceso a instalaciones de este tipo, además de que es un uso complementario a la nueva instalación de zonas educativas.

- Extensión administrativa del ayuntamiento y oficinas. Para conseguir un aumento de puestos de trabajo en esta zona y, secundariamente, un aumento en la mezcla de usos.

- Parque infantil de tráfico. Aumentar la mezcla de usos de la zona y dotar de un espacio verde que permita revitalizar una zona deprimida.

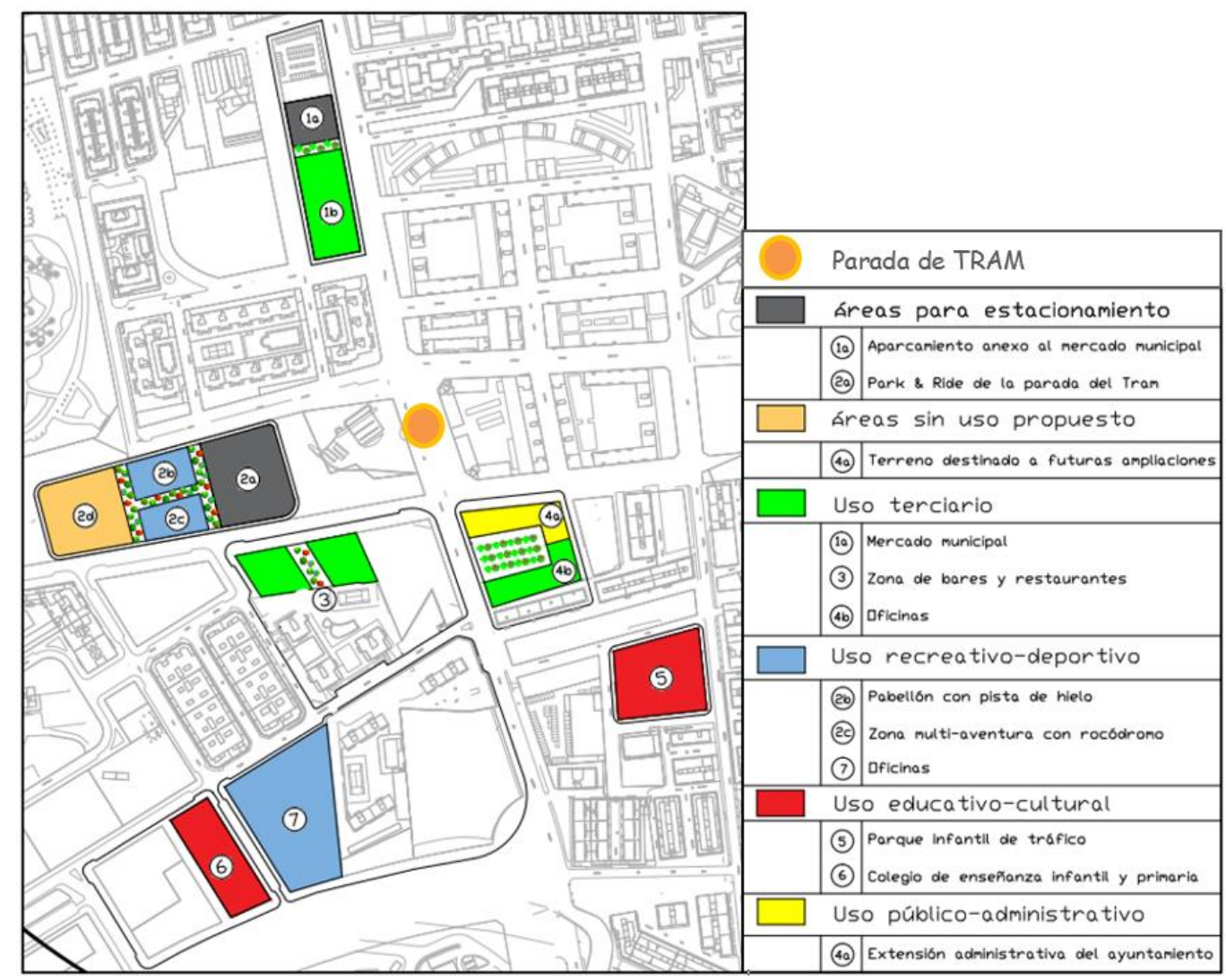

Fig. 6 - Desarrollo urbanístico propuesto en el área de influencia de la parada

\section{AGRADECIMIENTOS}

Los autores agradecen al Vicerrectorado de Investigación, Desarrollo e Innovación de la 
Universidad de Alicante la concesión de las ayudas enmarcadas en el Programa Propio para el fomento de la I+D+i, cuya beneficiaria es la coautora Patricia Fernández Aracil.

\section{REFERENCIAS}

AYUNTAMIENTO DE ALICANTE. (2007). Proyecto Urban. Barrios Zona Norte de Alicante.

BERTOLINI, L. (1999). Spatial Development Patterns and Public Transport: The Application of an Analytical Model in the Netherlands. Planning Practice \& Research, $\mathrm{N}^{\circ}$ 14(2), pp. 199-2010.

CENTER FOR TRANSIT-ORIENTED DEVELOPMENT. (2009). Five years of progress. CITY AND COUNTY OF DENVER. (2006). Community planning and development, Transit-Oriented Development Strategic Plan.

CURTIS, C., RENNE, J.L. y BERTOLINI, L. (2009). Transit Oriented Development. Making it Happen. Ashgate, Farnham/Burlington.

GIBSON, K. y ABBOTT, C. (2002). City profile: Portland, Oregon. Cities, Vol. 19, № 6, pp. 425-436.

KNOWLES, R.D. (2012). Transit Oriented Development in Copenhagen, Denmark: from the Finger Plan to Ørestad. Journal of Transport Geography, N² 22, pp. 251-261

IBEAS, A., GONZÁLEZ, F., DELL'OLIO, L. y MOURA, J.L. (2007). Manual de Encuestas de Movilidad. Preferencias Reveladas. Universidad de Cantabria, Santander.

MINISTERIO DE AGRICULTURA, ALIMENTACIÓN Y MEDIO AMBIENTE. (2010). Sistema Municipal de Indicadores de Sostenibilidad.

ORTUÑO, A. (2013). El modelo "Transit-Oriented Development (TOD)". Posibilidades de implementación en el corredor Alicante-Benidorm. Revista Iberoamericana de urbanismo, $\mathrm{N}^{\mathrm{o}} 10$, pp. 23-33.

PORTAL WEB DE CONSULTA DE LA ENCUESTA DE INFRAESTRUCTURA Y EQUIPAMIENTOS LOCALES, EIEL. (2015). http://eiel.diputacionalicante.es/

RECONNECTING AMERICA. (2009). Case Studies for Transit-Oriented Development. Briefing Report Number 3. Reconnecting America, Washington, DC.

REUSSER, D.E., LOUKOPOULOS, P., STAUFFACHER, M., y SCHOLZ, R.W. (2008). Classifying railway stations for sustainable transitions - balance node and place functions. Journal of transport geography, № 16, pp. 191-202.

VECTALIA SUBUS. (2015). http://www.subus.es/ 